\title{
Moving Toward Precision Medicine in Psoriasis and Psoriatic Arthritis
}

\author{
Christopher T. Ritchlin (i), Stephen R. Pennington (1D, Nick J. Reynolds (D), \\ and Oliver FitzGerald (D)
}

\begin{abstract}
Current management approaches for the treatment of psoriasis and psoriatic arthritis (PsA) are imprecise and depend largely on clinical assessment. A more precise approach, which takes into account an individual patient's variations in genes, proteins, environment, and lifestyle, is beginning to receive attention with the most advanced progress seen in the treatment of cancer. Herein, the methodological approaches required for this precision medicine approach to be adopted in psoriatic disease, as well as their advantages, are reviewed. In addition, advances that are being made to address areas of unmet need in PsA, notably the use of proteomic approaches, are presented with suggestions that combine genetic and protein data (proteogenomics). Finally, progress that is being made in 2 large-scale, multipartner studies focused on the development of a precision medicine approach to the treatment of skin psoriasis is presented and discussed. (J Rheumatol Suppl. 2020 June;96:19-24; doi:10.3899/jrheum.200122)
\end{abstract}

Key Indexing Terms:

PSORIATIC ARTHRITIS PSORIASIS

PRECISION MEDICINE

GRAPPA

From the Division of Allergy, Immunology, and Rheumatology,

University of Rochester Medical Center, Rochester, New York, USA; School of Medicine, Conway Institute of Biomolecular and Biomedical Research, University College Dublin (UCD), Dublin, Ireland; Institute of Translational and Clinical Medicine, Newcastle University and Newcastle Dermatology, Newcastle Hospitals National Health Service (NHS) Foundation Trust, Newcastle upon Tyne, UK; Department of Rheumatology, St. Vincent's University Hospital, Dublin, Ireland. As part of the supplement series GRAPPA 2019, this report was reviewed internally and approved by the Guest Editors for integrity, accuracy, and consistency with scientific and ethical standards.

NJR is a UK National Institute for Health Research (NIHR) Senior Investigator and is also supported by the Newcastle NIHR Biomedical Research Center, the Newcastle Medical Research Council (MRC) and the Engineering and Physical Sciences Research Council (EPSRC) Molecular Pathology Node, and the Newcastle NIHR Medtech and In vitro diagnostic Co-operative. NJR has received honoraria, travel support, and/or research grants (through Newcastle University) from AbbVie, Almirall, Celgene, Janssen, Novartis, Pfizer, and UCB Pharma Ltd. SRP is supported by Science Foundation Ireland and funding from the European Union's Horizon 2020 research and innovation program under grant agreement number 82574. The UCD Conway Institute is funded by the Programme for Research in Third Level Institutions as administered by the Higher Education Authority of Ireland.

C.T. Ritchlin, MD, MPH, Professor of Medicine, Division of Allergy, Immunology, and Rheumatology, University of Rochester Medical Center; S.R. Pennington, PhD, BSc, ARCS, Professor of Proteomics, School of Medicine, Conway Institute of Biomolecular and Biomedical Research, UCD; N.J. Reynolds, BSc, MB BS, MD, Professor of Dermatology, Institute of Translational and Clinical Medicine, Newcastle University and Newcastle Dermatology, Newcastle Hospitals NHS Foundation Trust; O. FitzGerald, MD, FRCPI, FRCP(UK), Newman Clinical Research Professor, Department of Rheumatology, St. Vincent's University Hospital, and Conway Institute for Biomolecular and Biomedical Research, UCD.

Address correspondence to Dr. O. FitzGerald, Newman Clinical Research Professor, Dept. of Rheumatology, St. Vincent's University Hospital, and Conway Institute for Biomolecular Research, University College Dublin, Belfield Dublin 4, Dublin, Ireland.E-mail: oliver.fitzgerald@ucd.ie
The current therapy selection process for patients with psoriasis and psoriatic arthritis (PsA) is imprecise and largely empirical. Many factors contribute to therapy selection, including a physician's experience with psoriasis and PsA treatment, as well as clinical phenotypic features, comorbidities, patient preferences, pharmaceutical marketing strategies, and financial or payor restraints. It is a difficult and perplexing experience for a patient who has active disease, because failure to respond adequately to treatment typically precipitates a cycle of therapy changes before a treatment is "stumbled upon" that results in a meaningful benefit.

The increasing development and success of biologic treatment for psoriasis has resulted in many patients achieving complete or almost complete skin responses [Psoriasis Area Severity Index (PASI) 100 or 90 responses, respective$1 y]^{1,2}$. Nevertheless, only a proportion of patients achieve such outcomes to the first prescribed biologic, and patients typically cycle through a number of agents before achieving an adequate response ${ }^{3,4}$. Moreover, for patients with PsA, the fairly low bar of the American College of Rheumatology (ACR) 20 response is met in 40-60\% of randomized controlled trials with disease-modifying antirheumatic drugs. Thus, more than $40 \%$ of patients do not respond according to current definitions, and those who do respond often still have substantial levels of active disease. Further, divergent responses are not uncommon, whereby one component of disease will respond but another disease component will not or will get worse. Together, these responses contribute to poorer short-term and longterm outcomes. Because of this,

Personal non-commercial use only. The Journal of Rheumatology Copyright $\subset$ 2020. All rights reserved. 
a better method of treatment selection needs to be developed; the treatment paradigm must move from a paradigm in which all patients appear to be equally likely to respond to all available treatments, to a treatment paradigm where, based on a careful assessment of a patient's clinical, genetic, and biomarker profile, a more rational treatment choice can be made.

Herein, Professor Christopher T. Ritchlin (USA) discusses how close we are to developing a precision medicine approach and reflects on the experience across the medical specialties. Next, Professor Stephen R. Pennington (Ireland) reviews progress related to the development of precision medicine approaches in psoriatic disease, predominantly PsA. Last, Professor Nick J. Reynolds (UK) discusses psoriasis and provides an update from the British Association of Dermatologists' Biologics and Immunomodulators Register (BADBIR) and Psoriasis Stratification to Optimise Relevant Therapy (PSORT) project.

\section{Precision Medicine: How Near Are We to Perfection?}

PsA is characterized by great complexity and heterogeneity. Complexity is observed in the interaction of several different cellular and molecular pathways that are present in multiple tissues, as well as the diversity of domain (skin, joints, spine, enthesitis, dactylitis) involvement that varies widely from patient to patient ${ }^{5}$. Heterogeneity is exemplified by the wide range and combination of skin and musculoskeletal phenotypes and the associated comorbidities that greatly complicate diagnosis and therapy. Indeed, despite the approval of 11 agents in the USA to treat PsA, therapeutic responses have not risen significantly when compared to outcomes reported in the early trials with anti-tumor necrosis factor agents over 15 years ago ${ }^{6}$. Thus, it is imperative that we develop new strategies to define the dominant pathophysiologic pathways and link basic scientific discoveries to key clinical variables. Fortunately, major technological advances over the past several years provide unparalleled opportunities to examine cellular and molecular pathways and interactions in the blood and tissue that provide the critical tools that are necessary to apply a precision medicine approach to the care of the patient with PsA.

Precision medicine is defined as "an emerging approach for disease treatment and prevention that takes into account individual variability in genes, environment, and lifestyle for each person"7. Combining precision and personalized medicine in the evaluation of the patient with PsA will allow us to fully address complexity and heterogeneity and apply a tailored therapeutic methodology. In this section, the technologies that are now available to define specific cell subpopulations, the importance of examining target tissues in addition to sampling the blood, and the required steps to convert the electronic medical record (EMR) from a billing document to a rich resource for the collection and analysis of patient-related data will be discussed.
The application of new technologies to the study of cells and signaling pathways in the blood and synovial tissues provides new insights into key pathophysiologic mechanisms and relevant effectors ${ }^{8}$. In particular, transcriptomic profiling of cells at the single cell (sc) level [sc RNA sequencing (seq)] has revealed that cells of the same lineage that express interleukin (IL)-17 express distinct mRNA profiles that differ dramatically in different target tissues from patient to patient ${ }^{9,10}$. Hence, data derived from flow cytometry or immunohistochemistry do not reveal the full complexity and heterogeneity of the pathologic process. Additional technologies are also providing a view of key pathologic events in patients with inflammatory arthritis, including ATAC-seq (Assay for Transposase-Accessible Chromatin using sequencing) for analysis of the epigenome and cytometry by time-of-flight (a type of flow cytometry that uses heavy metals instead of fluorochromes to label antibodies). These technologies have greatly expanded the number of surface markers that can be examined. The readout for this technology is by mass spectrometry (MS).

CITE-seq (Cellular Indexing of Transcriptomes and Epitopes by sequencing), a method that connects RNA seq with qualitative and quantitative data on surface proteins at the sc level, is a promising technology that provides a more comprehensive picture through the simultaneous analysis of both RNA and protein expression. The functional topology of cells in target tissues can now be understood through the use of Slide-seq to study cellular transcriptomes on tissue sections and laser capture microdissection, in which specific cells of interest are isolated and analyzed from microscopic regions of tissues. It is also important to note that the cost of the new technologies is declining owing to the development of low-cost alternative methodologies ${ }^{11}$.

Various publications emphasize that understanding key pathologic events in inflammatory arthritis requires the analysis of cells and pathways in the synovial tissue that are often not revealed by sampling the peripheral blood. For example, scRNA analysis of rheumatoid synovium revealed subsets of Thy1+ and Thy1-fibroblastoid cells with distinct locations in the synovial membrane and that these subsets are characterized by discrete effector functions such as tissue degradation and the release of inflammatory cytokines ${ }^{12}$. It has also been demonstrated that specific Type 17 cells are sequestered in the synovium and not the blood in $\operatorname{Ps}^{13}$. Subsets that are of interest as key effectors in PsA include $\mathrm{CD} 8+$ resident memory $\mathrm{T}$ cells ${ }^{14}$, gamma delta $\mathrm{T}$ cells $\mathrm{s}^{15}$, and monocytes with surface markers linked to effector function $^{16}$. It is anticipated that the study of synovium, skin, and blood from patients with psoriatic disease will foster a precision medicine strategy that can be individualized to a single patient. Of course, such studies will require tissue sampling from the synovium as well as the skin, and it is likely that the technologies for safe and rapid sampling of synovial tissue will also evolve rapidly.

Personal non-commercial use only. The Journal of Rheumatology Copyright $(\subset) 2020$. All rights reserved. 
Detailed phenotypic and outcomes data are critical to place the findings of blood and tissue analysis in context. Currently, EMR collect an abundance of relevant patient information, but they are primarily organized for coding and billing. Efforts are under way to better organize and analyze this vast trove of data to improve patient care ${ }^{17}$. The current datasets will require better categorization, which is complicated by the need to integrate formats and structures from a number of different sources ${ }^{18}$. Ontogenies, which define relationships between concepts that can foster logical reasoning, will need to be developed. These efforts will be catalyzed by applied statistical methods and machine learning. Moreover, the availability of wearable health devices and biomonitoring will provide a rich source of patient data that can help advance diagnostics and therapeutics.

Precision medicine in the current era is far from perfection. Yet we are witnessing a revolution in technologies that reveal detailed information on molecular and cellular processes in the blood and tissue that will shape diagnostic and therapeutic strategies with high specificity. These efforts will be amplified by the availability of high-quality phenotypic and outcomes data to inform treatment decisions on individual patients. It is anticipated that this revolution will greatly alter the diagnostic and therapeutic landscape in psoriasis and PsA in the near future.

\section{Applying Precision Medicine to Psoriatic Disease}

Precision medicine is an emerging approach to disease treatment and prevention that considers individual variability in genes, environment, and lifestyle. Currently, rheumatologists tailor their clinical treatment decisions to detailed clinical assessment and individual patient factors that include age, sex, patient preferences, locally available resources, preexisting conditions, comorbidities, and other mitigating circumstances. Precision medicine represents a new frontier for improving patient outcomes in psoriatic disease that combines data generated by genomics with other omics, including proteomics and metabolomics. Using "big data" analytics with machine-learning and artificial intelligence approaches, an individual's molecular and clinical data can be analyzed against a background of population-level data.

While significant and impressive advances are being made in some disease areas (notably oncology), other specialties are seemingly adopting these new molecular, data-driven strategies in a more measured (slower) approach . To date, the implementation of precision medicine has relied largely on genomic data. While this is undoubtedly important, it represents just one component of the complex interplay between genes and environment that contributes to an individual's clinical presentation and response to therapeutic intervention(s). As the reliability and throughput of other omics technologies improve the quality and quantity of the data they can generate, they will increasingly be included in data-driven precision medicine. Indeed, as long ago as
2004, Leroy Hood, who was responsible for the first gasphase protein sequencer (1982), DNA synthesizer (1983), peptide synthesizer (1984), and automated DNA sequencer (1986), began to introduce the concept of Predictive, Preventive, Personalized, and Participatory (P4) ${ }^{19}$ medicine. In it, systems medicine big data would be analyzed by methods created in the digital revolution, and when combined with social networks, deliver a new era of P4 medicine ${ }^{20}$. Combining genomic with proteomic data, 1 form of "proteogenomics" is set to revolutionize precision medicine, and again, it seems that oncology leads the way ${ }^{21}$. Because proteins are the main molecular executors of biological processes, Hood and Flores suggest that, of all the omics, proteomics has the greatest potential to improve the health of individuals ${ }^{20}$.

Biomarkers, which are measurable indicators of disease (current or future status), play a key role in the delivery of healthcare and the development of new omics biomarkers that will underpin the delivery of precision medicine. At present, many diagnostic tests rely on the measurement of individual proteins in blood. It has been argued that newer diagnostics will comprise signatures of multiple proteins and other analytes that, when combined with relevant clinical data, will support precision medicine. Clearly, the heterogeneity of PsA represents an ideal opportunity for the development of multiplexed protein biomarker signatures, including those that might help stratify treatment and identify changes in disease activity with treatment.

\section{Key Areas of Unmet Need in PsA}

The Group for Research and Assessment of Psoriasis and PsA (GRAPPA) Collaborative Research Network has identified key areas of unmet need in PsA that include (1) the identification of patients with psoriasis who will develop PsA, (2) the development of markers of PsA disease progression to guide therapies and prevent irreversible joint damage, and (3) the prediction of response to treatment in PsA and psoriasis.

(1) Identification of patients with psoriasis who will develop PsA. About $30 \%$ of patients with psoriasis develop PsA. This transition and progression are determined by a complex interplay of genes, microbiome, immune system modulation, and environmental exposures. Notably, from an epidemiological perspective, key etiologic risk factors have been identified. Subclinical changes in the entheses and joints have been investigated in psoriasis cases using ultrasound and magnetic resonance imaging to ascertain whether they predict progression to PsA. However, there are limited prospective data, and results are heterogeneous.

Again, the expectation is that new molecular markers will support the early identification of those patients who are progressing to PsA. It is established that HLA genotype modulates disease expression in PsA. This highlights the need for dense genotyping of immune-related susceptibil-

Personal non-commercial use only. The Journal of Rheumatology Copyright $(\subset) 2020$. All rights reserved. 
ity loci and the emerging and compelling evidence for the existence of PsA-specific risk loci ${ }^{22}$. While HLA-B27 is the best available genetic marker of progression to PsA in patients with skin psoriasis, whether susceptibility loci identified as being specific to PsA are sufficiently sensitive and/or specific to discriminate those who may develop PsA from those with psoriasis without PsA is still debatable. Combining protein markers with data on HLA haplotype may support the more precise and accurate early detection of PsA in patients with psoriasis.

(2) PsA disease progression to irreversible joint damage. Known clinical prognostic markers of progression to joint damage include polyarticular disease, dactylitis, high acute-phase response, and delayed presentation to specialist rheumatology care. Joint erosions and damage are markers of severe disease and have previously been demonstrated to be associated with poorer functional status, higher economic effects, and a risk factor for mortality. The GRAPPA PsA-BioDAM project was developed with the primary objective of determining the independent predictive ability of serum biomarkers for joint damage, with the hypothesis that surrogate protein biomarkers will predict which patients are at risk for peripheral radiographic damage in PsA.

(3) Prediction of response to treatment in PsA and psoriasis. There remains a major unmet need for biomarkers to predict treatment response in PsA and psoriasis. In oncology, disease management is now molecularly guided to treatments that are less likely to be toxic and more likely to be efficacious with resultant improved clinical outcomes. This "personalized" and "precise" approach, which is tailored to the individual patient's genetic, epigenetic, cellular, and/or molecular phenotype, is much needed in psoriatic disease management. The current treatment paradigm in psoriatic disease is imprecise. Treatment choices may lead to poorly controlled disease, and ultimately to progressive structural damage. A precision medicine approach in psoriatic disease could provide a more rational stepwise approach; speedier achievement and maintenance of treat-to-target goals of remission or low disease activity; better prediction of an adverse effect profile; reduced toxicity; less structural damage; better work productivity; and "guided regimes" for treatment maintenance, tapering, or withdrawal.

In ongoing work, Professors Pennington and Oliver FitzGerald have combined data from several previous studies $^{22,23,24,25}$ to develop the Protein Biomarker Assays for PsA (PAPRICA), which allows for the simultaneous measurement of over 200 candidate blood protein biomarkers. This assay works by using a powerful targeted proteomics strategy, in which multiple proteins known to have an association with a particular disease (such as PsA) are measured simultaneously by targeting peptides (fragments of proteins). The measurement technology exploits peptide separation by liquid chromatography (LC) and precise, sensitive, and quantitative detection of peptides by MS, called LC/MS .
The specificity of the method - knowing which peptides are measured - is assured by fragmenting the peptides in the mass spectrometer and measuring the peptide fragments in a state-of-the-art technique known as multiple reaction monitoring (MRM). Targeted proteomics was recognized as Nature Methods magazine's Method of the Year in $2012^{26}$. The University College Dublin (UCD) team published one of the first multiplexed protein MRM assays in $2006^{27}$, an assay that is now commercially available from international reagent and laboratory tool provider Merck-Sigma.

The PAPRICA biomarker panel is undertaken using a blood serum sample that, after a preparation process that UCD has streamlined, is analyzed on a triple quadrupole mass spectrometer. The data from the measurement of the multiple peptides is in turn analyzed by machine-learning approaches, including random forests and Bayesian Additive Regression Trees $^{28}$.

PAPRICA has several key advantages, including that it is minimally invasive (liquid biopsy), has $200+$ blood protein biomarkers that can be measured simultaneously from a $2-\mu \mathrm{L}$ blood (serum) sample, allows proteins to be measured precisely in a triple quad mass spectrometer, allows data to be combined and analyzed by machine learning, and has a sample-to-sample assay time of $72 \mathrm{~min}$. The development of the PAPRICA assay and other complementary strategies has the capacity to underpin the development of more effective decision-making tools for a precision medicine approach for PsA.

\section{Toward Precision Medicine in Psoriasis: Update from the BADBIR and PSORT Programs}

The World Health Organization 2016 report on psoriasis recommended that patients should have access to "comprehensive, individually adapted treatment," which is essentially personalized or precision medicine (ifpa-pso.com/ our-actions/advocacy/who-global-report). Although great advances have been made with the development of biologics and new small-molecule agents, many therapeutic challenges remain. These include how best to use the therapeutic agents that are available to cost-effectively induce disease clearance, and importantly, to maintain remission across all disease phenotypes and severities. Precision or stratified medicine is based on the idea that there are subpopulations within a disease category (e.g., chronic plaque psoriasis) that can be identified using biomarkers, preferably at baseline (prior to therapeutic intervention) or during the early phases of therapy. Such biomarkers can be used to predict clinical response to specific drugs (and/or adverse events) and thereby guide the choice of the most suitable therapeutic agent for particular subjects. These concepts have been primarily developed in the cancer field but are now being applied to a range of inflammatory disorders, as illustrated below in 2 UK-based studies, BADBIR and PSORT.

Linked to the identification of these subpopulations is the

Personal non-commercial use only. The Journal of Rheumatology Copyright $(\subset) 2020$. All rights reserved. 
concept of disease and drug endotype ${ }^{29}$. The concept of disease endotype implies that a clinical label such as psoriasis is an umbrella term, beneath which sit subgroups that can be defined by genetics and molecular mechanisms that associate with specific clinical phenotypes or drug pharmacology. One example is generalized pustular psoriasis, in which anti-IL-1 and anti-IL-36 drugs are predicted on the basis of underlying pathophysiological mechanisms, to block the abnormal signaling pathway in this subset of patients. Indeed, early-phase trials have shown dramatic improvement within days of receiving a single intravenous dose of a monoclonal antibody against the IL-36 receptor, which was not dependent on the presence of the IL-36RN mutation ${ }^{30}$. This elegant study provides evidence that molecular definition of a psoriasis disease endotype informs precise drug targeting.

BADBIR is a prospective, longitudinal, observational study of patients with psoriasis who started or switched to biologic, conventional, or small-molecular systemic therapy within the previous 6 months ${ }^{31}$. To date, BADBIR has enrolled about 17,000 participants, about one-third of whom have contributed to the bioresource. Several publications have emanated from BADBIR that are relevant to the concepts of precision medicine. For example, overall, $77 \%$ of subjects starting a biologic or switching from one biologic to another continue taking this biologic for at least 1 year, reinforcing the effectiveness of biologics in real-life practice $^{3,4}$. However, there were clear differences in effectiveness (measured as persistence on therapy) between different biologics ${ }^{3,4}$. Moreover, subjects experiencing treatment failure with one biologic therapy benefited from switching to another ${ }^{4}$. For example, as compared to adalimumab (ADA), etanercept was a predictor for discontinuation, whereas ustekinumab was a predictor for drug persistence in both biologic-naive subjects and those switching biologics. Notably, multiple comorbidities predicted withdrawal after switching. A number of demographic and clinical factors were associated with reduced odds of achieving $\geq 90 \%$ improvement in PASI at 6 months: female sex $(0.78$, 95\% CI 0.66-0.93), social status (such as unemployment), current or former smoking history, psoriasis of the palms and/or soles, and the presence of small plaques [only compared with small and large plaques $(0.78,95 \% \mathrm{CI}$ $0.62-0.96)]^{32}$. On the other hand, white ethnicity and higher baseline PASI were associated with increased odds of achieving PASI 90. These types of clinical variables are being built into ongoing studies that are aiming to derive biomarkers and to construct an algorithm for testing in clinical practice.

PSORT is a multidisciplinary consortium, comprising a number of UK academic institutions, life science companies, and patient groups. Its principal aims are to investigate disease and drug endotype in psoriasis and derive clinically relevant and scalable biomarkers to stratify response to biologic therapy. PSORT's integrative structure comprises 2 main workstreams with appropriately powered discovery, refinement, and validation cohorts. The discovery cohort comprises 82 deeply phenotyped subjects who were starting biologic therapy (ustekinumab or ADA) in routine clinical practice. Blood and skin samples were collected at prespecified intervals on patients at baseline through Week 12 . Ongoing analyses include drug levels, flow cytometry/mass cytometry, DNA for genotyping, and RNAseq of both skin and blood samples. PSORT has placed significant emphasis on building up the data layer by layer, as well as data integration and health economic analysis. To date, PSORT has derived normal serum drug ranges for ADA and ustekinumab and has shown that early drug levels of ADA predict clinical outcome at 6 months into treatment ${ }^{33,34}$. It has also shown that HLA-C*0602-negative patients are more likely to respond to ADA (measured as PASI 90 clinical response $)^{35}$. Ultimately, PSORT aims to derive a multifaceted algorithm that integrates clinical RNAseq and genetic data, as well as serum drug levels that have been shown to be predictive of response in a recent study of $\mathrm{ADA}^{33}$. These studies provide increased evidence that a better understanding of disease and drug endotypes will allow for the clinical application of precision medicine.

There are many challenges, though, and these efforts will require an integrative systems approach. These efforts will also require innovative analytical frameworks to deal with the high-dimensional datasets and to derive scalable algorithms for testing in clinical settings. To succeed, there is a need to develop and champion academic and industrial partnerships as well as international collaborative networks.

\section{DISCUSSION}

Herein, the need for the development of a precision medicine approach to the treatment of patients with psoriasis and PsA is discussed. Such an approach would replace the current inaccurate method whereby successful treatment is more often stumbled upon rather than chosen through a carefully crafted treatment algorithm. It is expected that a precision medicine approach will have several benefits, including a patient's increased ability to participate in work and leisure activities. As well, a reduction is expected in the effect of the disease on levels of pain, in overall treatment cost, and in functional impairment that results from joint damage. Given several recent technological advances, the time is now appropriate to apply these approaches to psoriasis and PsA in academic and industrial partnerships as well as international collaborative networks.

\section{REFERENCES}

1. Reich K, Armstrong AW, Langley RG, Flavin S, Randazzo B, $\mathrm{Li}$ S, et al. Guselkumab versus secukinumab for the treatment of moderate-to-severe psoriasis (ECLIPSE): results from a phase 3, randomised controlled trial. Lancet 2019;394:831-9.

2. Reich K, Gooderham M, Thaci D, Crowley JJ, Ryan C, Krueger JG, et al. Risankizumab compared with adalimumab in patients with

Personal non-commercial use only. The Journal of Rheumatology Copyright $(\subset) 2020$. All rights reserved. 
moderate-to-severe plaque psoriasis (IMMvent): a randomised, double-blind, active-comparator-controlled phase 3 trial. Lancet 2019;394:576-86.

3. Warren RB, Smith CH, Yiu ZZN, Ashcroft DM, Barker JNWN, Burden AD, et al. Differential drug survival of biologic therapies for the treatment of psoriasis: a prospective observational cohort study from the British Association of Dermatologists Biologic Interventions Register (BADBIR). J Invest Dermatol 2015;135:2632-40.

4. Iskandar IY, Warren RB, Lunt M, Mason KJ, Evans I, McElhone K, et al. Differential drug survival of second-line biologic therapies in patients with psoriasis: observational cohort study from the British Association of Dermatologists Biologic Interventions Register (BADBIR). J Invest Dermatol 2018;138:775-84.

5. Ritchlin CT, Colbert RA, Gladman DD. Psoriatic arthritis. N Engl J Med 2017;376:957-70.

6. Alten R, Conaghan PG, Strand V, Sullivan E, Blackburn S, Tian $\mathrm{H}$, et al. Unmet needs in psoriatic arthritis patients receiving immunomodulatory therapy: results from a large multinational real-world study. Clin Rheumatol 2019;38:1615-26.

7. Biro K, Dombradi V, Jani A, Boruzs K, Gray M. Creating a common language: defining individualized, personalized and precision prevention in public health. J Public Health 2018;40:e552-e9.

8. Mimitou EP, Cheng A, Montalbano A, Hao S, Stoeckius M, Legut M, et al. Multiplexed detection of proteins, transcriptomes, clonotypes and CRISPR perturbations in single cells. Nat Methods 2019;16:409-12.

9. Kishi Y, Kondo T, Xiao S, Yosef N, Gaublomme J, Wu C, et al. Protein $\mathrm{C}$ receptor (PROCR) is a negative regulator of Th17 pathogenicity. J Exp Med 2016;213:2489-501.

10. Stadhouders R, Lubberts E, Hendriks RW. A cellular and molecular view of T helper 17 cell plasticity in autoimmunity. J Autoimmun 2018;87:1-15.

11. Stephenson W, Donlin LT, Butler A, Rozo C, Bracken B, Rashidfarrokhi A, et al. Single-cell RNA-seq of rheumatoid arthritis synovial tissue using low-cost microfluidic instrumentation. Nat Commun 2018;9:791.

12. Croft AP, Campos J, Jansen K, Turner JD, Marshall J, Attar M, et al. Distinct fibroblast subsets drive inflammation and damage in arthritis. Nature 2019;570:246-51.

13. Menon B, Gullick NJ, Walter GJ, Rajasekhar M, Garrood T, Evans $\mathrm{HG}$, et al. Interleukin-17+CD8+ T cells are enriched in the joints of patients with psoriatic arthritis and correlate with disease activity and joint damage progression. Arthritis Rheumatol 2014; 66:1272-81.

14. Matos TR, O’Malley JT, Lowry EL, Hamm D, Kirsch IR, Robins $\mathrm{HS}$, et al. Clinically resolved psoriatic lesions contain psoriasis-specific IL-17-producing alphabeta T cell clones. J Clin Invest 2017;127:4031-41.

15. Nguyen CT, Maverakis E, Eberl M, Adamopoulos IE. Gammadelta $\mathrm{T}$ cells in rheumatic diseases: from fundamental mechanisms to autoimmunity. Semin Immunopathol 2019;41:595-605.

16. Culemann S, Gruneboom A, Nicolas-Avila JA, Weidner D, Lammle $\mathrm{KF}$, Rothe T, et al. Locally renewing resident synovial macrophages provide a protective barrier for the joint. Nature 2019;572:670-5.

17. Parikh RB, Schwartz JS, Navathe AS. Beyond genes and molecules - a precision delivery initiative for precision medicine. N Engl J Med 2017;376:1609-12.

18. Haendel MA, Chute CG, Robinson PN. Classification, ontology, and precision medicine. N Engl J Med 2018;379:1452-62.

19. Weston AD, Hood L. Systems biology, proteomics, and the future of health care: toward predictive, preventative, and personalized medicine. J Proteome Res 2004;3:179-96.
20. Hood L, Flores M. A personal view on systems medicine and the emergence of proactive $\mathrm{P} 4$ medicine: predictive, preventive, personalized and participatory. N Biotechnol 2012;29:613-24.

21. Rodriguez H, Pennington SR. Revolutionizing precision oncology through collaborative proteogenomics and data sharing. Cell 2018;173:535-9

22. Ademowo OS, Hernandez B, Collins E, Rooney C, Fearon U, van Kuijk AW, et al. Discovery and confirmation of a protein biomarker panel with potential to predict response to biological therapy in psoriatic arthritis. Ann Rheum Dis 2016;75:234-41.

23. Collins ES, Butt AQ, Gibson DS, Dunn MJ, Fearon U, van Kuijk AW, et al. A clinically based protein discovery strategy to identify potential biomarkers of response to anti-TNF-alpha treatment of psoriatic arthritis. Proteomics Clin Appl 2016;10:645-62.

24. Gohar F, McArdle A, Jones M, Callan N, Hernandez B, Kessel C, et al. Molecular signature characterisation of different inflammatory phenotypes of systemic juvenile idiopathic arthritis. Ann Rheum Dis 2019;78:1107-13.

25. Mc Ardle A, Flatley B, Pennington SR, FitzGerald O. Early biomarkers of joint damage in rheumatoid and psoriatic arthritis. Arthritis Res Ther 2015;17:141.

26. Method of the year 2012. Nat Methods 2013;10:1.

27. Jenkins RE, Kitteringham NR, Hunter CL, Webb S, Hunt TJ, Elsby $\mathrm{R}$, et al. Relative and absolute quantitative expression profiling of cytochromes $\mathrm{P} 450$ using isotope-coded affinity tags. Proteomics 2006;6:1934-47.

28. Hernandez B, Raftery AE, Pennington SR, Parnell AC. Bayesian Additive Regression Trees using Bayesian model averaging. Stat Comput 2018;28:869-90.

29. Eyerich K, Brown SJ, Perez White BE, Tanaka RJ, Bissonette R, Dhar S, et al. Human and computational models of atopic dermatitis: a review and perspectives by an expert panel of the International Eczema Council. J Allergy Clin Immunol 2019;143:36-45

30. Bachelez H, Choon SE, Marrakchi S, Burden AD, Tsai TF, Morita A, et al. Inhibition of the interleukin-36 pathway for the treatment of generalized pustular psoriasis. N Engl J Med 2019;380:981-3.

31. Burden AD, Warren RB, Kleyn CE, McElhone K, Smith CH, Reynolds NJ, et al; BADBIR Study Group. The British Association of Dermatologists' Biologic Interventions Register (BADBIR): design, methodology and objectives. Br J Dermatol 2012; 166:545-54.

32. Warren RB, Marsden A, Tomenson B, Mason KJ, Soliman MM, Burden AD, et al; BSTOP study group; PSORT consortium. Identifying demographic, social and clinical predictors of biologic therapy effectiveness in psoriasis: a multicentre longitudinal cohort study. Br J Dermatol 2019;180:1069-76.

33. Wilkinson N, Tsakok T, Dand N, Bloem K, Duckworth M, Baudry D, et al. Defining the therapeutic range for adalimumab and predicting response in psoriasis: a multicenter prospective observational cohort study. J Invest Dermatol 2019;139:115-23.

34. Tsakok T, Wilson N, Dand N, Loeff FC, Bloem K, Baudry $\mathrm{D}$, et al; British Association of Dermatologists Biologic and Immunomodulators Register (BADBIR) Study Group and the Psoriasis Stratification to Optimise Relevant Therapy (PSORT) Consortium. Association of serum ustekinumab levels with clinical response in psoriasis. JAMA Dermatol 2019 Sep 18 (E-pub ahead of print).

35. Dand N, Duckworth M, Baudry D, Russell A, Curtis CJ, Lee SH, et al; BADBIR Study Group; BSTOP Study Group; PSORT Consortium. HLA-C*06:02 genotype is a predictive biomarker of biologic treatment response in psoriasis. J Allergy Clin Immunol 2019;143:2120-30. 